\title{
Research on Robot Vision System Based on Image Model Feature Extraction
}

Jie Zhang*

Dongguan University of Technology, Dongguan 523808, China

\begin{abstract}
Algorithm encryption of wavelet image which has excellent localization property in time domain and frequency domain can locate graphic information having different directional characteristics into any precision level in certain extent. It has feature matched with human visual characteristics because of the disappearance of blocking effect and the noise. The article first analyzes the wavelet transformation principle of image model in feature extraction process and the second iteration process. Dynamic image feature extraction model can be established using this method in robot vision system. The result of envelope is more realistic and the effect is obvious. The application of the diversity training to project and contrast, the comparison precision is high and relative error is low.
\end{abstract}

Keywords: Image model, Feature extraction, Robot vision, Wavelet transform.

\section{INTRODUCTION}

Computer graphic technology widely rose in developed countries in the 1980s. With the development of computer graphics technology in China, it began their new life in China. Image model extraction of computer graphic technology through the computer to wipe off the noise, enhance, restore, divide and extract the feature. There are differences and similarities between the basic two-dimensional spline and the three-dimensional basic shape of the modeling. Computer graphics model extraction can regulate the color, material, density and other factors of the entire machine visual design through the computer directly easily which improves the quality and speed of design and saves manpower and resources and receives the glorify of the designers. Core image coding of the image model extraction can compress image signal through transform, wavelet transform, neural networks, model based and other coding and reduce the amount of data storage, the amount of data and the transmission bandwidth. The amount of information can be compressed to analyze and identify the image. Image analysis can turn image described by pixel into simpler description of the target using edge detection, segmentation, feature extraction and other means.

\section{MODEL PRINCIPLE OF IMAGE MODEL FEA- TURE EXTRACTION}

The most commonly used model of the image feature extraction is the wavelet transform function model which mainly processed in color, shape, surface smoothness, the positional relationship, image features and other sub-modules. Match and retrieval of the characteristics of different image files can be expressed through the following function relationship. Assuming that $\psi(t) \in L^{2}(R) L^{2}(R)$ is histogram of the image file and system database. It can transfer into $\hat{\psi}(\omega)$ using Fourier transform. $\psi(t)$ represents a basis wave. The similarity of two images can be translated through Euclidean distance which process is shown as follows.

From the Fig. (1), we can get the wavelet sequence which is shown as follows:

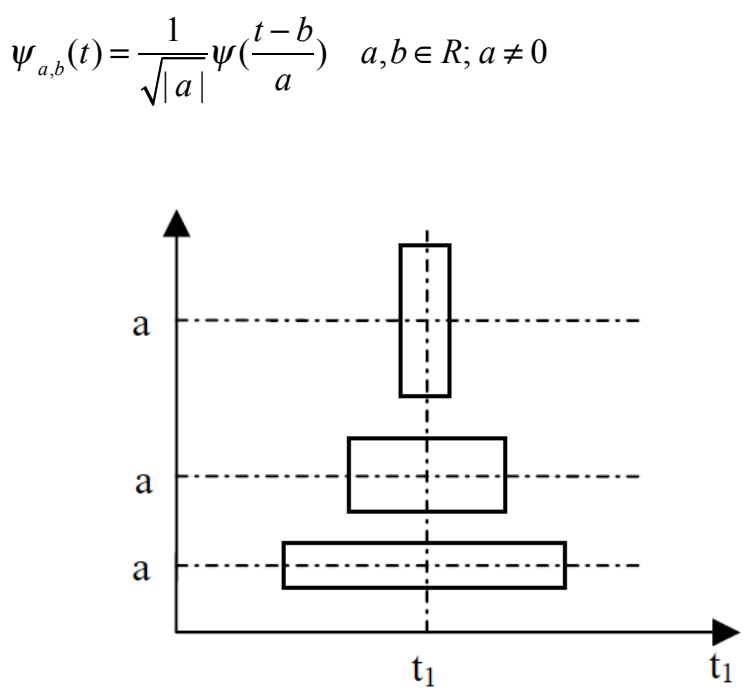

Fig. (1). Time-frequency window of wavelet transform.

After the image retrieval, the similarity of all histogram is $\phi(x)$ and $\psi(x)$, its expression is:

$$
\phi(x)=\sum_{n} h_{\phi}(n) \sqrt{2} \phi(2 x-n)
$$




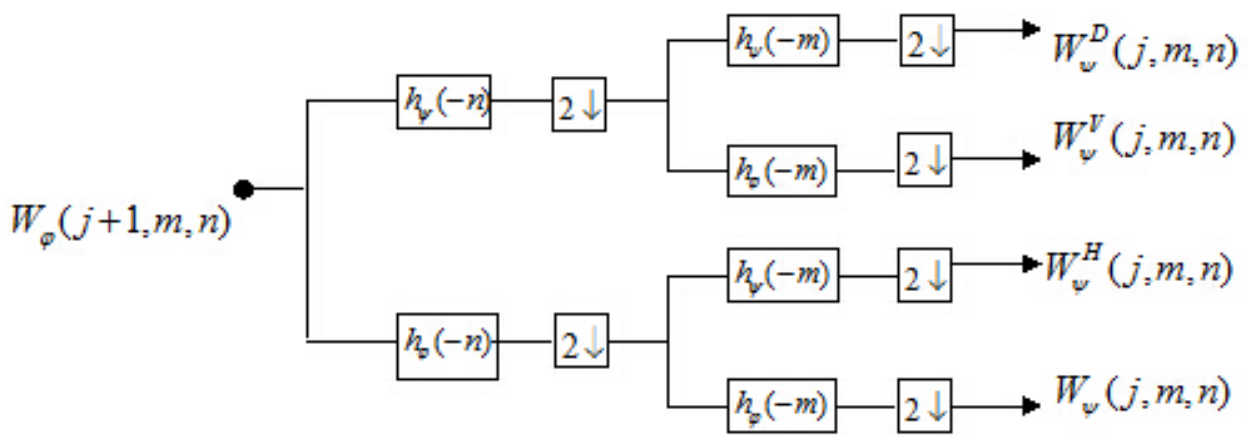

Fig. (2). Image secondary iterative process.

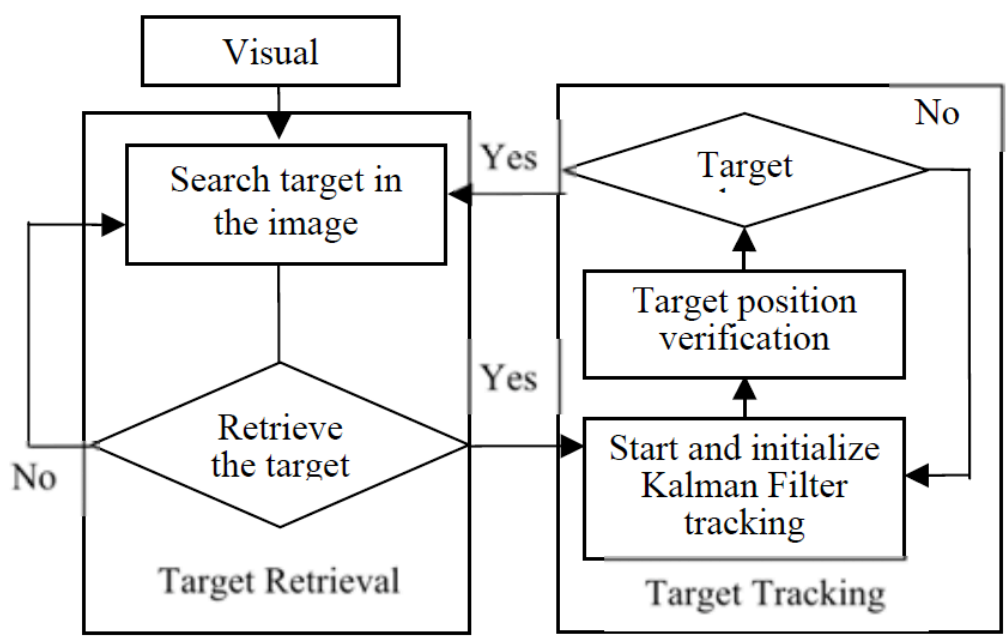

Fig. (3). Frame diagram of image feature extraction of robot vision system.

$\psi(x)=\sum_{n} h_{\psi}(n) \sqrt{2} \psi(2 x-n)$

In the above formula, $h_{\phi}$ and $h_{\psi}$ is the vector and vectorscale wavelet of similarity, the secondary iterative process using wavelet is shown as follows:

In Fig. (2), if $U_{j}^{0}=V_{j}, \quad U_{j}^{1}=W_{j}, j \in Z$, decomposition relationship can be expressed as:

$U_{j+1}^{0}=U_{j}^{0}+U_{j}^{1}, \quad j \in Z$

In the extraction process of the image, for the more resolution, $\varphi(t)$ and $\psi(t)$ meet the two-scale equation:

$$
\begin{aligned}
& \varphi(t)=\sum_{k \in z} h_{k} \varphi(2 t-k)\left\{h_{k}\right\}_{k \in z} \in l^{2} \\
& \psi(t)=\sum_{k \in z} g_{k} \psi(2 t-k)\left\{g_{k}\right\}_{k \in z} \in l^{2}
\end{aligned}
$$

In the above formula, $u_{0}(t)$ is scaling function $\varphi(t)$ and $u_{1}(t)$ is wavelet basis function $\psi(t) .\left\{u_{n}(t)\right\}\left(n \in Z^{+}\right)$can constitute orthogonal wavelet packet ensured by basis function $u_{0}(t)$. For colorful image, the collection of the sample of image file is the transform and expression of brightness or color information into discrete values. For the true performance of the original image file, intervals of sample should be selected appropriately.

\section{IMAGE FEATURE EXTRACTION OF ROBOT VI- SION SYSTEM}

The principle of robot vision system image feature extraction is the detection and tracking of the target. Image position of the target in robot vision system image feature extraction model can be ensured through detection and tracking which specific framework is shown as follows.

As is shown in Fig. (3), target tracking not only needs the characterization information of the target, but also needs the dynamics principle to match and locate the timeline of the target. Because the tracking cannot determine the initial position of the target, target detection is needed to determine the initial position of the target which can improve the efficiency and accuracy of shot detection. But, the machine has been 
level $\mathbf{i}+1$
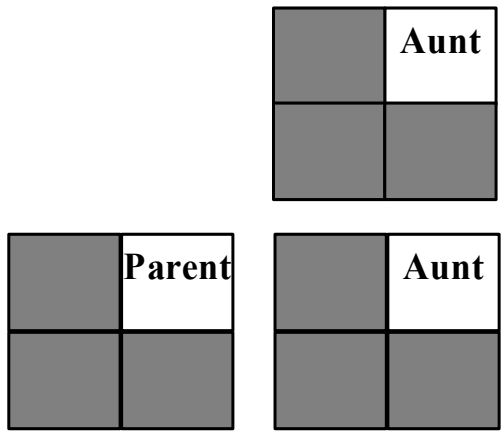

V

D

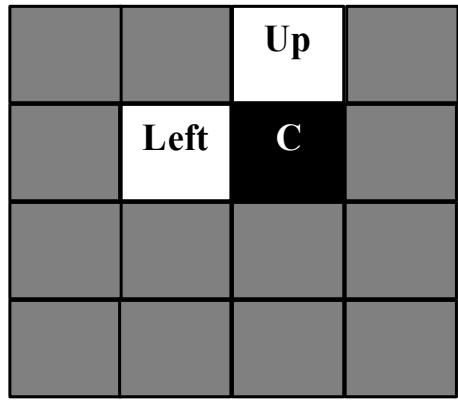

Vertical

Fig. (4). Computing schematic of need target block area.

occluded when it is in the process of motion, sometimes the track will failed. In order to reduce errors and the occlusion problem, the article presents a calculation method which is shown as follows:

As is shown in Fig. (4), apparent matching criterion of foreground and shield background can be extracted using SSD apparent characteristics. In the actual process, we can build goal and background the two templates in the same time. When we investigate any one area, we can do the related optimal matching of the two templates. $\left\{u_{n}(t)\right\}$ is the wavelet packet family of $\{h(k)\}$. We can use the following way to generate subspace family. Assuming that $n=1,2$, $\ldots ; j=1,2, \ldots$, if we use wavelet packet to decompose subspace $U_{j}^{n}$, there is:

$W_{j}=U_{j}^{1}=U_{j-1}^{2}+U_{j-1}^{3}, \quad j \in Z$

$U_{j-1}^{2}=U_{j-2}^{4} \oplus U_{j-2}^{5}, U_{j-1}^{3}=U_{j-2}^{6} \oplus U_{j-2}^{7}$

Decomposition formula can be got through iterative decomposition. If $n$ is octave refinement parameter and $n=2^{l}+m$, wavelet packet can be simplified as:

$\psi_{j, k, n}(t)=2^{-j / 2} \psi_{n}\left(2^{-j} t-k\right)$

\section{level i}

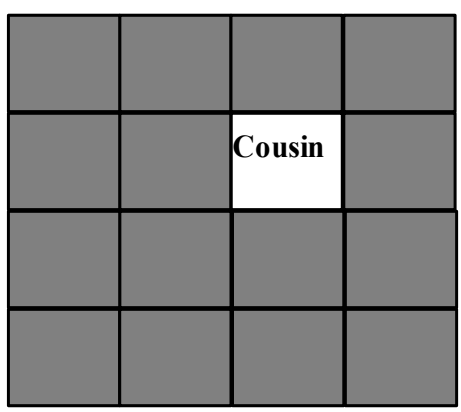

Horizontal

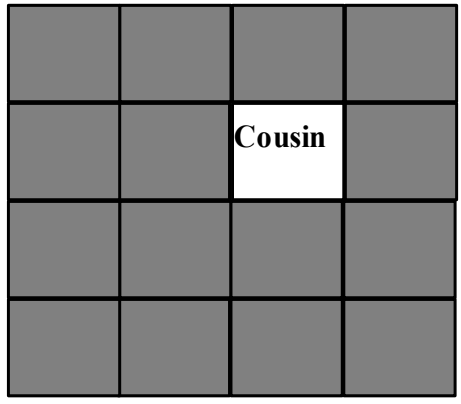

Diagonal

In this formula, $\psi_{n}(t)=2^{-l / 2} u_{n}\left(2^{-l} t\right)$. We can call $\psi_{j, k, n}(t)$ as wavelet packet which has scale indicator $j$, position indicators $k$ and frequency indicators $n$. Envelope extraction result is shown as follows.

As is shown in Fig. (5), according to the standard specification of the image format, aspect ratio of a pixel is 1:1 which has been called a square lattice. This is a basic mode of image file that number - analog conversion commonly used. In some particular image file process, pixel lattice is not used as cardinal number sometimes. Digital converter can be realized through the control of the aspect ratio of the image file and the total number of pixels in the image vertically and horizontally and the effect is obvious.

\section{EMPIRICAL ANALYSIS OF EXPERIMENTAL RE- SULT}

In the process of image feature extraction of robot vision system, there is positive relationship between sample of image information and the maximum frequency of the image signal. According to Nyquist, frequency of information sampling should be three times greater than the maximum frequency of the image signal. Taking the NTS for example, its maximum frequency of image signal is $3.8 \mathrm{MHz}$ and sam algorithm of wavelet packet is shown as follows. Assuming 

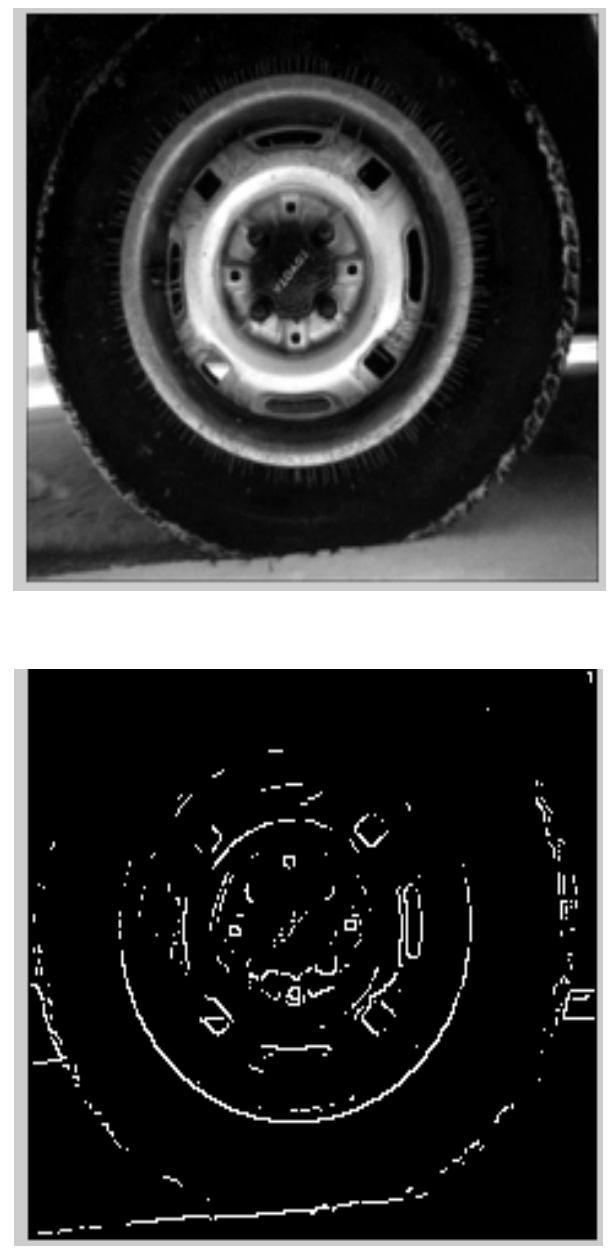

Fig. (5). Comparison charts of envelope extraction results.

that $g_{j}^{n}(t) \in U_{j}^{n}, g_{j}^{n}$ can be expressed as:pling frequency of signal is $7.6-10.4 \mathrm{MHz}$. Decomposition

$g_{j}^{n}(t)=\sum_{l} d_{l}^{j, n} u_{n}\left(2^{j} t-l\right)$

Wavelet packet decomposition algorithm can be expressed as $d_{l}^{j+1, n}, d_{l}^{j, 2 n}$ and $d_{l}^{j, 2 n+1}$ :

$d_{l}^{j, 2 n}=\sum_{k} a_{k-2 l} d_{k}^{j+1, n}$

$d_{l}^{j, 2 n+1}=\sum_{k} b_{k-2 l} d_{k}^{j+1, n}$

Based on the above formula, first, 400 image files of robot vision system should be virtualized and transferred the image information into digital image information. Number analog converter scan a virtual image from left to right, top to bottom and sample image information got from scanning and get voltage data information of each pixel. As is shown in the following Figure of 400 image model extraction training samples and 200 training samples, 'o' is normal image. ' + ' is stegoimage, thick line represents the classification threshold value T.
As is shown in Fig. (6), after the quantification of image file, image information data we got is an enormous value. We need use image coding techniques to simplify the information and reduce running stress of computer. Image coding technique is important assurance of the implement of the image file in the computer. The final result of experimental comparison is shown as follows.

We can enter and sum the feature model of image through weight level using this model and couple with selfload signal. According to the threshold of pixel characteristic, two functional units need to be operated. In the iterative process, it reduced with exponentially law. The model completed the choice of visual characteristics and the relevant characteristic has been enhanced and irrelevant feature has been ignored.

\section{CONCLUSION}

In the current image processing, the extraction of image element used weighted average method and the image pyramid method according to pixel level or feature extraction stage and multi-level image fusion. Image fusion of this method is not clear which has damage on the characteristics of the Information in the image. 


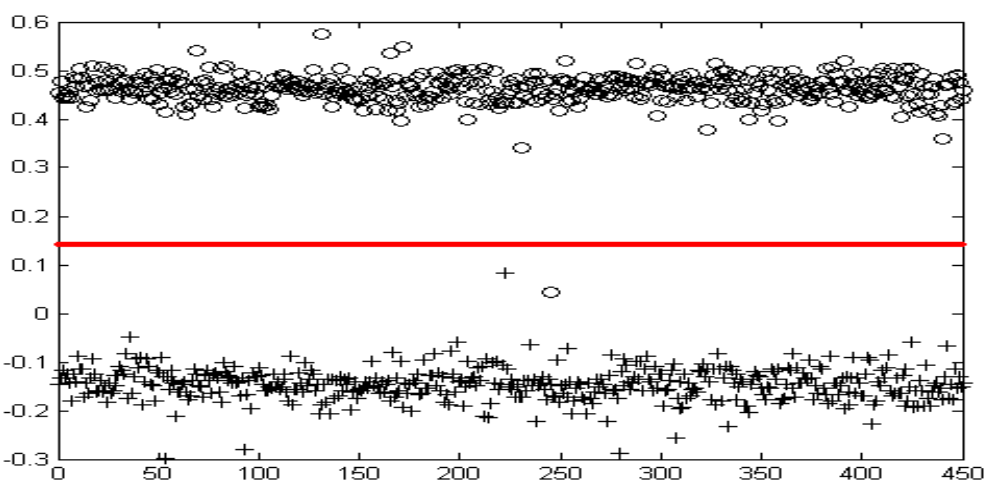

(a) 200 papers

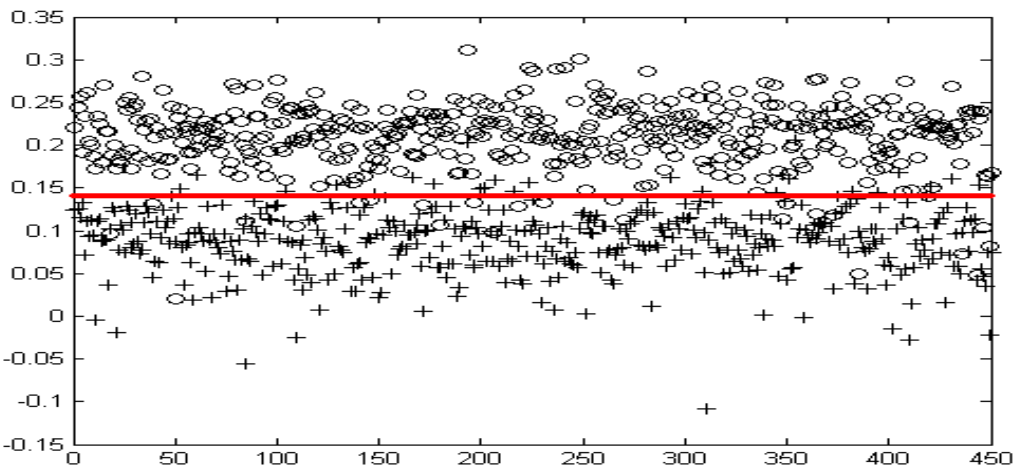

(b) 400 papers

Fig. (6). Comparison chart of feature extraction of training samples are projected of robot vision system.

Table 1. Comparison table of experimental result.

\begin{tabular}{|c|c|c|c|c|c|c|c|}
\hline \multirow{2}{*}{$\begin{array}{l}\text { Steganography } \\
\text { Tool }\end{array}$} & \multirow{2}{*}{$\begin{array}{l}\text { Embed } \\
\text { Amount }\end{array}$} & \multicolumn{2}{|c|}{ Farid } & \multicolumn{2}{|c|}{$\begin{array}{c}\text { The Absolute Value of the Wavelet } \\
\text { Packet Coefficients }\end{array}$} & \multicolumn{2}{|c|}{ Wavelet Packet Histogram } \\
\hline & & rN (\%) & rS (\%) & rN (\%) & rS (\%) & rN (\%) & rS (\%) \\
\hline \multirow{3}{*}{ F5 } & $4 \mathrm{k}$ & 96.365 & 69.029 & 97.981 & 79.271 & 100 & 85.596 \\
\hline & $5 \mathrm{k}$ & 97.201 & 72.486 & 98.019 & 80.554 & 100 & 87.439 \\
\hline & $6 \mathrm{k}$ & 97.567 & 78.157 & 98.213 & 83.016 & 100 & 88.168 \\
\hline \multirow{3}{*}{ Jsteg } & $4 \mathrm{k}$ & 97.357 & 70.881 & 98.367 & 73.417 & 100 & 89.849 \\
\hline & $5 \mathrm{k}$ & 97.918 & 73.774 & 98.829 & 76.688 & 100 & 87.271 \\
\hline & $6 \mathrm{k}$ & 98.012 & 76.024 & 98.911 & 80.767 & 100 & 89.646 \\
\hline \multirow{2}{*}{ OutGuess } & $4 \mathrm{k}$ & 96.921 & 68.813 & 98.832 & 78.853 & 100 & 89.871 \\
\hline & $5 \mathrm{k}$ & 97.230 & 69.627 & 98.901 & 79.634 & 100 & 90.227 \\
\hline
\end{tabular}

\section{CONFLICT OF INTEREST}

The authors confirm that this article content has no conflicts of interest.

\section{ACKNOWLEDGEMENTS}

This work is supported by the Scinece and Technology Project of Colleges and Institutes of Science Research of Dongguan(No.2012108102010). 


\section{REFERENCES}

[1] Chun Chen. Computer image processing technology and algorithms [M]. Tsinghua University Press, 2013.

[2] Xiaoxia Zheng, Weijian Li. Image Retrieval technology based on texture feature extraction $[\mathrm{J}]$. Heilongjiang Institute of Engineering (Natural Science Edition) .2005, December, Volume 19, No. 4

[3] Yepeng Guan, Weikang Gu. Research on the self-extraction algorithm of two-dimensional image feature points [J]. Sensing Technology Journal .2004, March, Section 1

[4] Fabien A. P. Petitcolas, Ross J. Anderson and Markus G. Kuhn. Information hiding- a survey". Proceedings of the IEEE, special issue on protection of multimedia content, July 1999, 87(7): 10621078

[5] Fridrich J, Goljan M, Hogea D. Steganalysis of JPEG images: breaking the F5 algorithm. Proc. 5th Int'l Workshop Information Hiding. Springer-Verlag, Noordwijkerhout, the Netherlands, 2002. 310-323

[6] Siqi Han, Lei Wang. Summary of threshold value method of image segmentation [J] System Engineering and Electronics Volume 24, 6: $91-94$
[7] Shuang Liu. Research on threshold selection method and its algorithm implementation in image segmentation[J]. Computer Knowledge and Technology: 68-70

[8] Wufan Chen. Wavelet analysis and the application in the picture processing (First edition) [M] Beijing: Science Press, 2002:121-142

[9] Westfeld A and Pfitzmann A. Attacks on steganographic systems. Proc. 3rd Int'l Workshop Information Hiding. SpringerVerlag,2009, Dresden, Germany, 61-76

[10] Huiming Wang, Ping Shi. Extraction method of image texture features. Communication University of China (Natural Science) .2006, March, Vol 13, No. 1

[11] Hongcheng Zhou, Huiying Dong. Modified edge detection based on wavelet image $[\mathrm{N}]$ Shenyang University of Technology .2005, (4)

[12] Fudong Ye. Analysis of image fusion algorithm based on wavelet transform [J], Hubei Vocational and Technical College of Ecological Engineering, 2011,9 (1).

(C) Jie Zhang; Licensee Bentham Open.

This is an open access article licensed under the terms of the Creative Commons Attribution Non-Commercial License (http://creativecommons.org/licenses/by-nc/3.0/) which permits unrestricted, non-commercial use, distribution and reproduction in any medium, provided the work is properly cited. 